\title{
Suscetibilidade da soja a percevejos na fase anterior ao desenvolvimento das vagens
}

\author{
Beatriz Spalding Corrêa-Ferreira(1)
}

(1)Embrapa Soja, Caixa Postal 231, CEP 86001-970 Londrina, PR. E-mail: beatriz@cnpso.embrapa.br

\begin{abstract}
Resumo - O objetivo deste trabalho foi avaliar os efeitos das populações de percevejos Euschistus heros (F.) e Piezodorus guildinii (West.) nas lavouras de soja nos meses de novembro/dezembro, antes do aparecimento das vagens nas plantas. Foram realizados experimentos em casa de vegetação e no campo, comparando-se infestações no período vegetativo e reprodutivo da cultura. As plantas de soja, mesmo sob o ataque de pesadas infestações de percevejos (até 8 por planta), no período vegetativo e no florescimento, não sofreram reduções significativas no rendimento. A qualidade das sementes foi semelhante entre os tratamentos e entre as espécies. No estádio R4, o número médio de vagens chochas foi diretamente proporcional ao aumento do número de percevejos por planta. No campo, as plantas com até quatro percevejos por metro, no final do período vegetativofloração, tiveram rendimentos médios e qualidade de sementes semelhantes aos das plantas livres de insetos. No período R5-R6, para as duas espécies de percevejos, o número de grãos picados e inviabilizados cresceram, e os rendimentos médios decresceram com o aumento da população, tendo-se obtido, para a qualidade da soja, um dano duas vezes maior para a espécie $P$. guildinii em relação a E. heros. Infestações desses percevejos, na fase vegetativa-floração, não causam reduções no rendimento e na qualidade das sementes de soja.
\end{abstract}

Termos para indexação: Glycine max, Euschistus heros, Piezodorus guildinii, Pentatomidae, manejo integrado.

\section{Susceptibility of soybean stink bugs prior to pod development}

\begin{abstract}
The objective of this work was to evaluate the damages caused by stink bug populations of Euschistus heros (F.) and Piezodorus guildinii (West.) to soybean crop on november/december, prior to pod set. Greenhouse and field experiments were carried out comparing infestations at vegetative-flowering and pod development stages. Soybean plants at vegetative and flowering growth stages, even under severe bugs infestation (8 per plant) did not show significant yield reduction. Seed quality, was similar among population levels and bug species. At R4 stage, the number of seedless pods increased proportionally to the number of bugs per plant. In field experiment, yield and seed quality of soybean plants infested with up to four E. heros or P. guildinii adults per meter, at the end of the vegetative-flowering stage, were similar to bug-free plants. The number of bugpunctured and inviable seeds increased and the average yield decreased with the increase of population levels of both bug species, at the pod filling stage (R5-R6) infestation. In relation to the soybean quality, the damage caused by $P$. guildinii was twice bigger than that caused by the brown stink bug. Infestations of these stink bugs on the vegetative-flowering stage do not cause reduction on yield and quality of soybean seeds.
\end{abstract}

Index terms: Glycine max, Euschistus heros, Piezodorus guildinii, Pentatomidae, integrated management.

\section{Introdução}

Os percevejos sugadores de sementes são considerados, em vários países, como uma das pragas de maior importância para a cultura da soja (Todd \& Herzog, 1980; Panizzi \& Slansky Júnior, 1985; Corrêa-Ferreira \& Panizzi, 1999; Panizzi et al., 2000); as espécies Euschistus heros (F.), Piezodorus guildinii (West.) e
Nezara viridula (L.) são as mais abundantes no Brasil (Corrêa-Ferreira \& Peres, 2003). Alimentam-se pela inserção de seus estiletes em diferentes estruturas da planta; sugam, preferencialmente, as vagens, atingindo diretamente os grãos de soja. O impacto da alimentação dos percevejos pode causar perdas significativas no rendimento, na qualidade e no potencial germinativo da soja (Todd \& Tunipseed, 1974; Panizzi \& Slansky 
Júnior, 1985; Russin et al., 1988; Villas-Bôas et al., 1990; Brier \& Rogers, 1991; Panizzi et al., 2000; CorrêaFerreira \& Azevedo, 2002; Santos, 2003). Os percevejos são, também, responsáveis por reduções no teor de óleo, aumento na percentagem de proteínas e ácidos graxos livres nas sementes, e pela transmissão de patógenos e de distúrbios fisiológicos, como a retenção foliar que, normalmente, estão associados à atividade alimentar desses pentatomídeos (Panizzi et al., 1979; Villas-Bôas et al., 1990; Sosa-Gómez \& Moscardi, 1995; Boethel et al., 2000).

Em geral, a presença dos percevejos na cultura está diretamente relacionada à presença das vagens nas plantas, cujo período entre o começo da frutificação e o ponto de acúmulo máximo de matéria seca no grão é o de maior sensibilidade da soja ao ataque desses insetos sugadores (Vicentini \& Jimenez, 1977; Shumann \& Todd, 1982; Brier \& Rogers, 1991; Gazzoni, 1998; Panizzi et al., 2000). Entretanto, muitas vezes esses pentatomídeos iniciam a colonização das lavouras no final do período vegetativo e início da floração. Nessa época, os percevejos saem da diapausa ou de hospedeiros alternativos e migram para a soja, aumentando progressivamente suas populações durante a fase reprodutiva (Corrêa-Ferreira \& Panizzi, 1999).

O efeito da alimentação dos percevejos sugadores, durante a fase reprodutiva da soja, foi intensivamente estudado e avaliado para diferentes espécies (Panizzi et al., 1979; Villas-Bôas et al., 1990; Gazzoni, 1998; Corrêa-Ferreira \& Azevedo, 2002). Entretanto, para o período anterior ao aparecimento das vagens, a literatura disponível é escassa, antiga e se refere especialmente à espécie P. guildinii (Galileo \& Heinrichs, 1978a, 1978b; Panizzi et al., 1979). Ao longo dos anos, verificaram-se grandes mudanças no sistema de produção de soja no Brasil e, paralelamente, mudanças também ocorreram com as populações de percevejos presentes nessa cultura; hoje, o percevejo marrom E. heros é a espécie mais abundante do complexo de pentatomídeos, especialmente do norte do Estado do Paraná ao Centro Oeste brasileiro (Corrêa-Ferreira \& Panizzi, 1999). Nessa região, essa espécie e o percevejo verde pequeno P. guildinii colonizam os campos de soja mesmo no mês de dezembro.

A ocorrência de elevadas populações desses percevejos, na fase inicial do desenvolvimento da cultura, e a falta de conhecimentos atuais disponíveis na literatura geram questionamentos e preocupação constante por parte de técnicos e produtores sobre seus possíveis da- nos e levam, muitas vezes, a um uso inadequado de produtos químicos altamente tóxicos para o seu controle.

Em conseqüência da diversidade de espécies de percevejos, presentes na cultura da soja, estudos comparativos foram realizados em diferentes áreas produtoras. Na Argentina, segundo Vicentini \& Jimenez (1977), P. guildinii é o percevejo que tem maior capacidade de causar danos à soja, apesar de não ser o responsável pelas maiores perdas de produção, em função da maior abundância do percevejo verde $N$. viridula presente naquela região. Resultados semelhantes foram constatados no Brasil por Corrêa-Ferreira \& Azevedo (2002) e Santos (2003), que confirmaram o maior potencial do P. guildinii de prejudicar a qualidade da semente de soja, em comparação às espécies $N$. viridula e E. heros, este último é responsável pelos menores danos. Em estudos comparativos, $N$. viridula causou dano semelhante a Acrosternum hilare (Say) nos Estados Unidos (Miner, 1961), e um dano três vezes mais severo que Piezodorus hybneri (Gmelin) na Austrália (Brier, 1993). Das espécies de percevejos sugadores mais comuns em soja no Brasil, $N$. viridula apresentou a maior frequiência alimentar em vagens verdes ou maduras (Santos, 2003). Para essa espécie de pentatomídeo, Panizzi \& Alves (1993) constataram que vagens de soja sem sementes (R3-R4), ou com sementes em início de desenvolvimento (R5), são fontes de alimento inadequado para as ninfas e adultos, quando comparados à soja em estágios mais avançados.

O objetivo deste trabalho foi avaliar os efeitos das populações de $E$. heros e $P$. guildinii, presentes nas lavouras de soja nos meses de novembro/dezembro, antes do aparecimento das vagens.

\section{Material e Métodos}

Para avaliar o dano causado à soja pelos percevejos $E$. heros e $P$. guildinii, no período inicial do desenvolvimento da cultura, experimentos em casa de vegetação (safra 2000/2001) e em campo (safra 2001/2002) foram instalados na Fazenda Experimental da Embrapa

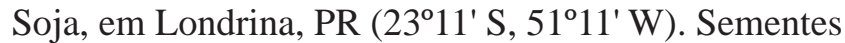
da cultivar Paraná foram semeadas em vasos e as plantas, cobertas com telas de filó, foram infestadas na fase vegetativa-floração, com adultos de E. heros e P. guildinii, por um período de 15 dias, nos níveis de 0 , 4 e 8 percevejos por planta (Ensaio 1). Paralelamente, nas plantas de soja da cultivar BR 37, comparou-se o 
dano de adultos de E. heros, causado no período vegetativo (V6), na floração (R1) e no final do desenvolvimento de vagens (R4). As plantas, em vasos, foram infestadas com 0, 2 e 4 percevejos por planta, durante 10 dias (Ensaio 2).

Em lavoura de soja com a cultivar BRS 134, utilizando-se gaiolas teladas (1x1x1 m), comparou-se o dano causado pelos percevejos, que colonizam a cultura antes do período de desenvolvimento de vagens (V9-R2), ao dano na época crítica de ataque desses insetos (R5-R6). As plantas de soja foram infestadas com adultos de $E$. heros e P. guildinii, por um período de 15 dias, nos níveis de 0,2 e 4 percevejos por metro linear (Ensaio 3).

Nos ensaios em casa de vegetação e no campo, utilizaram-se percevejos coletados em lavouras de soja. Para uniformizar a população dos percevejos a serem utilizados nas infestações, e reduzir o estresse da coleta, os percevejos foram mantidos por 24 horas em gaiolas, sob condições controladas, em salas de criação, antes de sua utilização. As plantas foram examinadas de dois em dois dias, com reposição dos percevejos mortos. Após o período de infestação, os percevejos foram eliminados manualmente (testes em casa de vegetação) ou com aplicação de endossulfan (no campo), e as plantas foram mantidas livres de insetos até a colheita. Avaliou-se o rendimento médio de grãos de soja, padronizado para $13 \%$ de umidade, o peso de sementes boas e a qualidade das sementes das plantas testemunhas e infestadas com percevejos. Na análise da qualidade das sementes, utilizou-se o exame visual em amostras de $50 \mathrm{~g}$, tendo-se classificado as sementes em três categorias: sementes boas (sementes sem dano aparente de percevejo, com cor e formatos normais), médias (sementes com algum dano) e ruins (sementes totalmente danificadas, enrugadas e chochas). Utilizou-se também o teste de tetrazólio, tendo-se avaliado as sementes picadas (Categoria 1-8) e as inviabilizadas pelo dano do percevejo (Categoria 6-8).

Nos ensaios, utilizou-se um delineamento inteiramente casualizado, com os tratamentos em esquema fatorial com seis (ensaio 1), sete (ensaio 2) e cinco (ensaio 3) repetições. Os dados foram submetidos à análise de variância, e as médias comparadas pelo teste de Tukey a $5 \%$ de probabilidade.

\section{Resultados e Discussão}

Quando se avaliou o dano causado à soja pelos percevejos que colonizaram a cultura antes do período de desenvolvimento de vagens - final do período vegetativo ao florescimento da soja (Ensaio 1) - verificou-se que as plantas de soja infestadas com até oito percevejos por planta, durante 15 dias, não tiveram seus rendimentos afetados, quando comparadas à produção das plantas livres de insetos (Tabela 1). Não foi constatada diferença significativa entre as espécies $E$. heros e P. guildinii ( $\mathrm{F}=3,23 ; \mathrm{GL}=1 ; \mathrm{p}<0,08)$, bem como entre os níveis testados ( $F=0,36 ; \mathrm{GL}=2 ; \mathrm{p}<0,70)$. $\mathrm{O}$ dano de percevejos na semente, avaliado pelo teste de tetrazólio, não demonstrou diferenças entre os tratamentos e entre as espécies testadas. Esses resultados obtidos para $E$. heros e P. guildinii confirmam aqueles obtidos para o percevejo verde pequeno, por Galileo \& Heinrichs (1978b) e Panizzi et al. (1979), e mostra que infestações de percevejos, nesse período de desenvolvimento da cultura, não geram danos qualitativos ou quantitativos, entretanto diferem dos obtidos por Nardi (2004). Esse autor, ao utilizar plantas de soja (cultivar CD 206) infestadas, por 39 dias (V5 ao R3), com três percevejos E. heros por planta, constatou que as alturas de plantas e os rendimentos foram menores do que o das plantas livres de insetos. Resultado que pode ser explicado pelo tempo elevado de exposição das plantas aos insetos e ao dano, durante o período de desenvolvimento de vagens (R3), quando as plantas são suscetíveis ao ataque dos percevejos sugadores (Galileo \& Heinrichs, 1978b; Panizzi et al., 1979).

Quando se comparou o dano causado por E. heros, durante 10 dias no período inicial do desenvolvimento da cultura (V6 e R1), com o final do período do desenvolvimento de vagens (R4) (Ensaio 2), obtiveramse rendimentos semelhantes para os índices de 0,2 e 4 percevejos por planta. Constatou-se, entretanto, que as plantas apresentaram uma redução gradual no peso

Tabela 1. Rendimento e qualidade de sementes de plantas de soja infestadas pelos percevejos colonizantes Euschistus heros e Piezodorus guildinii, durante 15 dias no período vegetativo-floração, em casa de vegetação (Ensaio 1).

\begin{tabular}{|c|c|c|c|}
\hline \multirow{2}{*}{$\begin{array}{l}\text { Percevejos } \\
\left(\mathrm{n}^{\mathrm{o}} \text { planta }^{-1}\right)\end{array}$} & \multirow{2}{*}{$\begin{array}{c}\text { Rendimento } \\
\left(\text { g planta }^{-1}\right)\end{array}$} & \multicolumn{2}{|c|}{ Qualidade $(\%)^{(1)}$} \\
\hline & & $\mathrm{TZ}(1-8)$ & TZ (6-8) \\
\hline & \multicolumn{3}{|c|}{ Euschistus heros } \\
\hline 0 & $19,0 \pm 1,2^{\text {ns }}$ & $0^{\mathrm{ns}}$ & 0 \\
\hline 4 & $18,5 \pm 1,0$ & 0 & 0 \\
\hline 8 & $17,6 \pm 0,8$ & $0,2 \pm 0,2$ & 0 \\
\hline & \multicolumn{3}{|c|}{ Piezodorus guildinii } \\
\hline 0 & $16,4 \pm 0,8$ & 0 & 0 \\
\hline 4 & $17,6 \pm 1,2$ & $0,4 \pm 0,3$ & 0 \\
\hline 8 & $16,8 \pm 0,8$ & $0,2 \pm 0,2$ & $0,2 \pm 0,2$ \\
\hline
\end{tabular}

${ }^{(1)}$ Teste de tetrazólio; TZ(1-8): porcentagem de sementes picadas por percevejo; TZ(6-8): porcentagem de sementes inviabilizadas

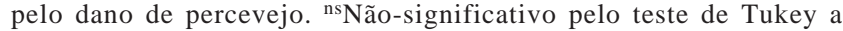
$5 \%$ de probabilidade. 
médio de sementes boas, que variou de 17,1 g (V6) para 13,2 g (R4), no nível de quatro percevejos por planta (Tabela 2). O peso médio de sementes boas de plantas atacadas por dois e quatro percevejos, no período vegetativo e na floração, foi estatisticamente igual ao das plantas livres de insetos. Só as plantas com infestações no final do desenvolvimento de vagens (R4) apresentaram produtividade significativamente menor, em relação às plantas testemunhas, o que confirma a suscetibilidade da soja aos danos dos percevejos, nessa fase do desenvolvimento (Panizzi et al., 1979, 2000; Brier \& Roger, 1991; McPherson et al., 1993; McPherson, 1996; Corrêa-Ferreira \& Panizzi, 1999; Santos, 2003; Nardi, 2004).

Embora seja conhecido que a presença de vagens chochas na planta de soja não ocorre somente por causa do dano de percevejos, quando se avaliou o número médio de vagens chochas, da parte superior das plantas de soja, constatou-se que os valores foram estatisticamente maiores, à medida que aumentou o número de percevejos por planta, no período do desenvolvimento de vagens (R4) (Tabela 3), que variou de 1,7 na testemunha para 9,1 vagens chochas, nas plantas com quatro

Tabela 2. Peso (g) de sementes boas de plantas de soja, infestadas por adultos de Euschistus heros durante 10 dias, em diferentes estádios fenológicos da planta, em casa de vegetação (Ensaio 2) ${ }^{(1)}$.

\begin{tabular}{lccc}
\hline Estádio de & \multicolumn{3}{c}{ Número de percevejos por planta } \\
\cline { 2 - 4 } infestação & 0 & 2 & 4 \\
\hline Vegetativo - V6 & $15,7 \pm 0,7 \mathrm{aA}$ & $14,8 \pm 1,3 \mathrm{aA}$ & $17,1 \pm 0,8 \mathrm{aA}$ \\
& $(89)^{(2)}$ & $(86)$ & $(92)$ \\
Floração - R1 & $15,7 \pm 1,0 \mathrm{aA}$ & $15 \pm 1,0 \mathrm{aA}$ & $14,8 \pm 2,0 \mathrm{aA}$ \\
& $(91)$ & $(95)$ & $(91)$ \\
Desenvolvimento & $19,8 \pm 0,9 \mathrm{aA}$ & $13,4 \pm 0,9 \mathrm{aB}$ & $13,2 \pm 1,7 \mathrm{aB}$ \\
de vagens - R4 & $(93)$ & $(83)$ & $(86)$ \\
\hline
\end{tabular}

(1)Médias seguidas pela mesma letra, minúscula na coluna e maiúscula na linha, não diferem entre si pelo teste de Tukey a 5\% de probabilidade. (2)Números entre parênteses são porcentuais médios de sementes boas (sementes sem dano causado por percevejo).

Tabela 3. Número médio de vagens chochas por planta, presentes na metade superior de plantas de soja, infestadas por adultos de Euschistus heros durante 10 dias, em diferentes estádios fenológicos da planta, em casa de vegetação (Ensaio 2)(1).

\begin{tabular}{lccc}
\hline Estádio de & \multicolumn{3}{c}{ Número de percevejos } \\
\cline { 2 - 4 } infestação & 0 & 2 & 4 \\
\hline Vegetativo - V6 & $2,7 \pm 1,0 \mathrm{aA}$ & $2,5 \pm 0,8 \mathrm{abA}$ & $2,4 \pm 0,8 \mathrm{bA}$ \\
Floração - R1 & $1,4 \pm 0,8 \mathrm{aA}$ & $1,3 \pm 0,8 \mathrm{bA}$ & $1,3 \pm 0,6 \mathrm{bA}$ \\
$\begin{array}{l}\text { Desenvolvimento } \\
\text { de vagens - R4 }\end{array}$ & $1,7 \pm 0,6 \mathrm{aC}$ & $5,3 \pm 1,3 \mathrm{aB}$ & $9,1 \pm 1,3 \mathrm{aA}$ \\
\hline
\end{tabular}

(1)Médias seguidas pela mesma letra, minúscula na coluna e maiúscula na linha, não diferem entre si pelo teste de Tukey a 5\% de probabilidade. percevejos. Plantas infestadas no período vegetativo ou na floração não apresentaram diferença entre os níveis de 0 , 2 e 4 percevejos por planta.

Os resultados obtidos no campo confirmaram aqueles constatados nos testes realizados em casa de vegetação, tendo comprovado que a presença das espécies de percevejos E. heros e P. guildinii nas plantas de soja, no período anterior ao desenvolvimento das vagens, não causou prejuízo ao rendimento das plantas e nem à qualidade das sementes. Plantas de soja infestadas com até quatro percevejos por metro, durante 15 dias - final do período vegetativo ao florescimento da soja (V9-R2) - tiveram rendimentos médios semelhantes aos das plantas livres de insetos (Tabela 4). Entretanto, quando as infestações ocorreram no período de enchimento de grãos (R5-R6), considerado crítico ao ataque dos percevejos, obtiveram-se, para as espécies analisadas, rendimentos médios que foram inversamente proporcionais ao aumento da população, embora diferenças significativas só tenham sido constatadas para E. heros com quatro percevejos por metro (476 g), em relação às plantas testemunhas (636 g) (Tabela 4). Embora não se tenha observado diferenças significativas no rendimento das plantas infestadas com P. guildinii, no período R5-R6, em outros parâmetros avaliados, como peso de sementes boas (Tabela 5) e qualidade das sementes (Tabela 6), essa diferença foi constatada, o que confirma o efeito negativo desses percevejos, quando se alimentam de soja no período de enchimento de grãos.

Para infestações de percevejos, na fase do desenvolvimento das plantas anterior ao aparecimento das vagens, o peso médio de sementes boas (sem dano) foi estatisticamente igual à testemunha, não tendo sido constatada diferença entre as espécies de percevejos e

Tabela 4. Rendimento médio de plantas de soja, submetidas ao ataque de percevejos por 15 dias, no período vegetativo-floração e no período de enchimento de grãos, em gaiolas de campo, na safra 2001/2002(1).

\begin{tabular}{|c|c|c|}
\hline \multirow{2}{*}{$\begin{array}{c}\text { Percevejos } \\
\left(\mathrm{n}^{\mathrm{o}} \mathrm{m}^{-1}\right)\end{array}$} & \multicolumn{2}{|c|}{ Rendimento médio $(\mathrm{g})$ por gaiola } \\
\hline & Euschistus heros & Piezodorus guildinii \\
\hline & \multicolumn{2}{|c|}{ Vegetativo-floração } \\
\hline 0 & $600 \pm 15 \mathrm{aA}$ & $574 \pm 28 \mathrm{aA}$ \\
\hline 2 & $544 \pm 22 \mathrm{aA}$ & $523 \pm 59 \mathrm{aA}$ \\
\hline 4 & $607 \pm 56 \mathrm{aA}$ & $566 \pm 30 \mathrm{aA}$ \\
\hline & \multicolumn{2}{|c|}{ Enchimento de grãos } \\
\hline 0 & $636 \pm 27 \mathrm{aA}$ & $578 \pm 29 \mathrm{aA}$ \\
\hline 2 & $523 \pm 37 \mathrm{abA}$ & $564 \pm 24 \mathrm{aA}$ \\
\hline 4 & $476 \pm 44 \mathrm{bA}$ & $514 \pm 48 \mathrm{aA}$ \\
\hline
\end{tabular}

(1)Médias seguidas pela mesma letra, minúscula na coluna e maiúscula na linha, para cada fase de desenvolvimento da cultura, não diferem entre si pelo teste de Tukey a 5\% de probabilidade. 
entre os níveis testados, com valores médios obtidos de 49,3 g e de 49,3 g para plantas infestadas por $E$. heros e P. guildinii, respectivamente (Tabela 5). Entretanto, infestações no período R5-R6, causaram reduções significativas no peso de sementes boas, quando atacadas por dois ou quatro percevejos, em relação às plantas testemunhas, que variaram de 49,0 g a 46,3 g nas plantas com ataques de E. heros, e de 49,1 g a 44,6 g para P. guildinii (Tabela 5), tendo-se constatado um dano significativamente maior nas plantas de soja atacadas com quatro P. guildinii, em relação a esse mesmo número de $E$. heros. O efeito daninho de P. guildinii já foi relatado no passado (Panizzi et al., 1979) e, mais recentemente, por Sosa-Gómez \& Moscardi (1995), quando avaliaram a capacidade diferencial de causar retenção foliar, confirmada por Corrêa-Ferreira \& Azevedo (2002) e Santos (2003), que destacaram o maior potencial dessa espécie em causar prejuízos às sementes de soja.

No campo, o dano dos percevejos na semente, avaliado pelo teste de tetrazólio, foi praticamente nulo, quando o ataque ocorreu no período inicial da cultura, não se tendo constatado tendências em relação aos tratamentos testados, o que é explicado pela fase do desenvolvimento em que as plantas estiveram sob o ataque desses insetos. Quando o ataque ocorreu no período de enchimento de grãos, obteve-se um aumento de grãos picados e inviabilizados pelos percevejos, que foi proporcionalmente crescente ao índice de infestação testado. O dano causado pela espécie $P$. guildinii foi duas vezes maior que o dano causado pelo percevejo marrom (Tabela 6).

Tabela 5. Peso médio (g $50 \mathrm{~g}^{-1}$ ) de sementes boas de plantas de soja, infestadas em campo por adultos de Euschistus heros e Piezodorus guildinii durante 15 dias, em diferentes estádios fenológicos da planta ${ }^{(1)}$.

\begin{tabular}{ccc}
\hline $\begin{array}{c}\text { Percevejos } \\
\left(\mathrm{n}^{\mathrm{o}} \mathrm{m}^{-1}\right)\end{array}$ & Euschistus heros & Piezodorus guildinii \\
\hline \multicolumn{3}{c}{ Vegetativo-floração } \\
0 & $49,2 \pm 0,2 \mathrm{aA}$ & $49,4 \pm 0,2 \mathrm{aA}$ \\
2 & $49,5 \pm 0,2 \mathrm{aA}$ & $49,5 \pm 0,1 \mathrm{aA}$ \\
4 & $49,2 \pm 0,2 \mathrm{aA}$ & $49,0 \pm 0,1 \mathrm{aA}$ \\
\hline Média & $49,3 \mathrm{aA}$ & $49,3 \mathrm{aA}$ \\
\hline \multicolumn{3}{c}{ Enchimento de grãos } \\
0 & $49,0 \pm 0,1 \mathrm{aA}$ & $49,1 \pm 0,1 \mathrm{aA}$ \\
2 & $47,2 \pm 0,5 \mathrm{bA}$ & $47,5 \pm 0,5 \mathrm{bA}$ \\
4 & $46,3 \pm 0,8 \mathrm{bA}$ & $44,6 \pm 0,4 \mathrm{cB}$ \\
\hline Média & $47,5 \mathrm{bA}$ & $47,1 \mathrm{bA}$ \\
\hline
\end{tabular}

(1)Safra 2000/2001; médias seguidas pela mesma letra, minúscula na coluna e maiúscula na linha, não diferem entre si pelo teste de Tukey a 5\% de probabilidade; sementes boas: sementes sem dano causado por percevejo.
Tabela 6. Porcentagem de sementes de soja picadas e inviabilizadas, pelo dano causado por espécies de percevejos às plantas infestadas durante 15 dias, no período vegetativo-floração e no enchimento de grãos, em gaiolas de campo $^{(1)}$.

\begin{tabular}{|c|c|c|c|c|}
\hline \multirow{2}{*}{$\begin{array}{l}\text { Percevejos } \\
\left(n^{0} m^{-1}\right)\end{array}$} & \multicolumn{2}{|c|}{ Euschistus heros } & \multicolumn{2}{|c|}{ Piezodorus guildinii } \\
\hline & TZ 1-8 & TZ 6-8 & TZ 1-8 & TZ 6-8 \\
\hline & \multicolumn{4}{|c|}{ Vegetativo-floração } \\
\hline 0 & 0 & 0 & 0 & 0 \\
\hline 2 & $0,2 \pm 0,2$ & 0 & 0 & 0 \\
\hline 4 & 0 & 0 & 0 & 0 \\
\hline & \multicolumn{4}{|c|}{ Enchimento de grãos } \\
\hline 0 & 0 & 0 & 0 & 0 \\
\hline 2 & $0,6 \pm 0,4$ & 0 & $2,0 \pm 0,3$ & $0,8 \pm 0,2$ \\
\hline 4 & $2,6 \pm 0,5$ & 0 & $4,8 \pm 0,6$ & $2,2 \pm 0,6$ \\
\hline
\end{tabular}

${ }^{(1)}$ Teste de tetrazólio; TZ(1-8): porcentagem de sementes picadas; $\mathrm{TZ}(6-8)$ : porcentagem de sementes inviabilizadas.

Os resultados obtidos, referentes à qualidade da semente, confirmam a maior capacidade do P. guildinii em causar danos qualitativos às sementes de soja, fato já relatado por Vicentini \& Jimenez (1977) na Argentina, e por Corrêa-Ferreira \& Azevedo (2002) e Santos (2003) para as populações de percevejos no Brasil. Para o índice de quatro percevejos por metro, constatou-se um valor de 2,2\% de sementes inviabilizadas nas plantas infestadas com P. guildinii, enquanto para aquelas infestadas com o percevejo marrom esse índice foi de $0 \%$ (Tabela 6). Os dados confirmam que a intensidade dos danos causados pelos percevejos está diretamente relacionada à população e ao estádio de desenvolvimento da planta.

\section{Conclusões}

1. Infestações dos percevejos $E$. heros e P. guildinii, na fase do desenvolvimento da soja anterior ao aparecimento das vagens, não causam reduções no rendimento e na qualidade das sementes.

2. Medidas de controle para os percevejos E. heros e P. guildinii, da fase final do período vegetativo ao florescimento da soja, não se justificam.

\section{Agradecimentos}

Aos pesquisadores da Embrapa Soja, Antônio R. Panizzi, Daniel R. Sosa Gómez e Décio L. Gazzoni, pela revisão e análise crítica do manuscrito. 


\section{Referências}

BOETHEL, D.J.; RUSSIN, J.S.; WIER, A.T.; LAYTON, M.B.; MINK, J.S.; BOYD, M.L. Delayed maturity associated with southern green stink bug (Heteroptera: Pentatomidae) injury at various soybean phenological stages. Journal of Economic Entomology, v.93, p.707-712, 2000

BRIER, H. Extent of bug damage in soybeans dependent on species. Northern Focus, p.4-5, 1993.

BRIER, H.; ROGERS, J. Susceptibility of soybean to damage by Nezara viridula (L.) (Hemiptera: Pentatomidae) and Riptortus serripes (F.) (Hemiptera: Alydidae) during three stage of pod development. Journal of Australian Entomological Society, v.30, p.123-128, 1991.

CORRÊA-FERREIRA, B.S.; AZEVEDO, J. Soybean seed damage by different species of stink bugs. Agriculture and Forest Entomology, v.4, p.145-150, 2002.

CORRÊA-FERREIRA, B.S.; PANIZZI, A.R. Percevejos da soja e seu manejo. Londrina: Embrapa-CNPSo, 1999. 45p. (Circular técnica, 24).

CORRÊA-FERREIRA, B.S.; PERES, W.A.A. Comportamento da população dos percevejos-pragas e a fenologia da soja. In: CORRÊAFERREIRA, B.S. (Org.). Soja orgânica: alternativas para o manejo dos insetos-pragas. Londrina: Embrapa Soja, 2003. p.27-32.

GALILEO, M.H.M.; HEINRICHS, E.A. Avaliação dos danos causados aos legumes de soja (Glycine max (L.) Merrill) por Piezodorus guildinii (Westwood, 1837) (Hemiptera, Pentatomidae), em diferentes níveis e épocas de infestação. Anais da Sociedade Entomológica do Brasil, v.7, p.33-39, 1978a.

GALILEO, M.H.M.; HEINRICHS, E.A. Efeito dos danos causados por Piezodorus guildinii (Westwood, 1837) (Hemiptera, Pentatomidae), em diferentes níveis e épocas de infestação, no rendimento de grãos de soja (Glycine max (L.) Merrill). Anais da Sociedade Entomológica do Brasil, v.7, p.20-25, 1978b.

GAZZONI, D.L. Efeito de populações de percevejos na produtividade, qualidade da semente e características agronômicas da soja. Pesquisa Agropecuária Brasileira, v.33, p.1229-1237, 1998.

McPHERSON, R.M. Relationship between soybean maturity group and the phenology and abundance of stink bugs (Heteroptera: Pentatomidae): impact on yield and quality. Journal of Entomology Science, v.31, p.199-208, 1996.

McPHERSON, R.M.; DOUCE, G.K.; HUDSON, R.D. Annual variation in stink bug (Heteroptera: Pentatomidae) seasonal abundance and species composition in Georgia soybean and its impact on yield and quality. Journal of Entomology Science, v.28, p.61-72, 1993.

MINER, F.D. Stink bug damage to soybeans. Arkansas Agricultural Experiment Station Farm Research, v.10, p.12, 1961.

NARDI, L.C. Danos do percevejo-marrom Euschistus heros (Fabr.) às plantas de soja, em duas épocas de infestação. 2004. 52p. Dissertação (Mestrado) - Universidade Estadual do Oeste do Paraná, Marechal Cândido Rondon.

PANIZZI, A.R.; ALVES, R.M.L. Performance of nymphs and adults of the southern green stink bug (Heteroptera: Pentatomidae) exposed to soybean pods at different phenological stages of development. Journal of Economic Entomology, v.86, p.1088-1093, 1993.

PANIZZI, A.R.; McPHERSON, J.E.; JAMES, D.G.; JAVAHERY, M.; McPHERSON, R.M. Stink bugs (Pentatomidae). In: SCHAEFER, C.W.; PANIZZI, A.R. (Ed.). Heteroptera of economic importance. Boca Raton, Florida, USA: CRC, 2000. p.432-434.

PANIZZI, A.R.; SLANSKY JUNIOR, F. Review of phytophagous pentatomids (Hemiptera: Pentatomidae) associated with soybean in the Americas. Florida Entomology, v.68, p.184-203, 1985.

PANIZZI, A.R.; SMITH, J.G.; PEREIRA, L.A.G.; YAMASHITA, J. Efeito dos danos de Piezodorus guildinii (Westwood, 1837) no rendimento e qualidade da soja. In: SEMINÁRIO NACIONAL DE PESQUISADE SOJA, 1., 1979, Londrina. Anais. Londrina: EmbrapaCNPSo, 1979. v.2, p.59-78.

RUSSIN, J.S.; LAYTON, M.B.; ORR, D.B.; BOETHEL, D.J. Stink bug damage to soybeans. La Agriculture, v.32, p.23-24, 1988.

SANTOS, C.H. dos. Suscetibilidade da soja, Glycine max (L.) Merr. aos danos causados por Nezara viridula (L.), Euschistus heros (Fabr.) e Piezodorus guildinii (West.) (Heteroptera: Pentatomidae) e Neomegalotomus parvus West. (Heteroptera: Alydidae). 2003. 91p. Tese (Doutorado) - Universidade Federal do Paraná, Curitiba.

SCHUMANN, F.W.; TODD, J.W. Population dynamics of the southern green stink bug (Heteroptera: Pentatomidae) in relation to soybean phenology. Journal of Economic Entomology, v.75, p.748753, 1982

SOSA-GÓMEZ, D.R.; MOSCARDI, F. Retenção foliar diferencial em soja provocada por percevejos (Heteroptera: Pentatomidae). Anais da Sociedade Entomológica do Brasil, v.24, p.401-404, 1995.

TODD, J.W.; HERZOG, D.C. Sampling phytophagous Pentatomidae on soybean. In: KOGAN, M.; HERZOG, D.C. (Ed.). Sampling methods in soybean entomology. New York: Springer-Verlag, 1980. p.438-478.

TODD, J.W.; TUNIPSEED, S.G. Effects of southern green stink bug damage on yield and quality of soybeans. Journal of Economic Entomology, v.67, p.421-426, 1974.

VICENTINI, R.; JIMENEZ, H.A. El vaneo de los frutos en soja. [Paraná, Argentina: Instituto Nacional de Tecnología Agropecuaria; Estación Experimental Agropecuaria], 1977. 30p. (INTA serie técnica, 47).

VILLAS-BÔAS, G.L.; GAZZONI, D.L.; OLIVEIRA, M.C.N. de; PEREIRA, N.P.; ROESSING, A.C.; FRANÇA NETO, J.B.; HENNING, A. Efeito de diferentes populações de percevejos sobre o rendimento e seus componentes, características agronômicas e qualidade de sementes de soja. Londrina: Embrapa-CNPSo, 1990. 43p. (Boletim de pesquisa, 1).

Recebido em 21 de dezembro de 2004 e aprovado em 19 de abril de 2005 